\title{
Violência contra idosos no ambiente doméstico: prevalência e fatores associados $(\mathrm{Recife} / \mathrm{PE})$
}

\author{
Violence against the elderly in the home environment: \\ prevalence and associated factors (Recife, State of Pernambuco)
}

Andrezza Marques Duque ${ }^{1}$

Márcia Carrera Campos Leal ${ }^{2}$

Ana Paula de Oliveira Marques ${ }^{1}$

Fernanda Maria Vieira Eskinazi ${ }^{1}$

Amanda Marques Duque ${ }^{1}$

${ }^{1}$ Departamento de Medicina Social, Centro de Ciências da Saúde, Universidade Federal de Pernambuco. Av. Prof. Moraes Rêgo s/n, Cidade Universitária. 50670-901 Recife PE. andrezza.duque@yahoo.com.br ${ }^{2}$ Programa de PósGraduação em Psicologia, Centro de Filosofia e Ciências Humanas, Universidade Federal de Pernambuco.
Abstract The scope of this paper was to determine the prevalence and factors associated with domestic violence against the elderly. It is a crosssectional study with 274 subjects, aged 60 years or more, of both sexes. Data were collected through interviews at home or in the USF based on a script structured in three parts: questionnaire with socio-demographic and bio-demographic information, two rating scales and a tool for identifying abuse. Among the respondents, 20.8\% reported having experienced at least one type of violence in their home environment. An association was revealed between those living with a greater number of individuals, among women and elderly people who are dependent for day-to-day activities. After applying the logistic regression model, only the variables of sex and family configuration were significantly associated, with evidence of greater frequency among those who lived with six or more residents and women. These findings highlight the magnitude and seriousness of the problem and point to the need for action to combat violence against the elderly.

Key words Abuse of the elderly, Domestic violence, Prevalence and associated factors
Resumo Este artigo teve como objetivo de determinar a prevalência e os fatores associados à violência doméstica contra pessoas idosas. Estudo de corte transversal com 274 sujeitos, com 60 anos ou mais, de ambos os sexos. Os dados foram coletados por meio de entrevistas nos domicílios ou nas USF a partir de roteiro estruturado em três blocos: questionário com informações biodemográficas e sociodemográficas; duas escalas de avaliação e; instrumento para identificação de violência. Entre os entrevistados, 20,8\% relataram ter sofrido pelo menos um dos tipos de violência em seu ambiente doméstico. Observou-se associação entre os que moravam com um número maior de indivíduos, entre as mulheres e idosos dependentes para as atividades instrumentais da vida diária. Após o modelo de regressão logística, apenas as variáveis sexo e arranjo familiar mostraram-se associadas significativamente, havendo evidência de maior ocorrência entre os que coabitavam com seis ou mais moradores e as mulheres. Estes achados evidenciam a magnitude e gravidade do problema e alerta para a necessidade de ações no combate à violência contra a pessoa idosa.

Palavras-chave Maus tratos ao idoso, Violência doméstica, Prevalência e fatores associados 


\section{Introdução}

O envelhecimento populacional é uma realidade mundial. No Brasil, ocorre em ritmo acelerado, acarretando modificações nas políticas sociais e constituindo-se em um dos grandes desafios da Saúde Pública.

Segundo a Organização Pan-Americana de Saúde ${ }^{1}$, o Brasil está entre os países que aumentam mais rapidamente o índice de envelhecimento e, de acordo com projeções para o ano de 2025, haverá 46 idosos para cada 100 pessoas menores de 15 anos.

Veras $^{2}$ argumenta que essa nova realidade impulsiona a ocorrência de redefinições nas políticas de saúde voltadas à população idosa no que diz respeito à atenção às doenças de caráter crônico e às ações e estratégias de prevenção e atenção integral no cuidado à saúde do idoso.

No panorama atual, as transformações vivenciadas em decorrência do processo de envelhecimento populacional acelerado acarretam mudanças no perfil epidemiológico brasileiro. A esse contexto somam-se as novas formas de arranjos domiciliares e o estresse da vida moderna, proporcionando à família, ao Estado e à sociedade grandes desafios a serem enfrentados ${ }^{3}$.

O processo de envelhecimento populacional vem ocasionando uma gradual reorganização da estrutura familiar. Diante disto, torna-se necessária a criação de um ambiente onde a velhice não seja percebida de maneira negativa e as necessidades do idoso possam acompanhar o ritmo e estilo de vida dos demais integrantes da família ${ }^{4}$.

Acrescente-se a esses fatores o empobrecimento da população, a invalidez física ou mental do idoso, um padrão de vida prévio permeado pela relação de violência, o estresse do cuidador, seus problemas e dificuldades, a moradia conjunta, as perdas materiais, o isolamento social, a doença do idoso e a consequente diminuição de sua capacidade funcional e cognitiva ${ }^{3}$.

A violência contra o idoso é objeto de estudo recente; segundo Bruno ${ }^{4}$ e Espíndola e Blay ${ }^{5}$, as publicações sobre os maus-tratos na terceira idade mostram-se ainda incipientes, sobretudo em relação à população brasileira. Entretanto, 0 acelerado crescimento desse segmento etário torna-se um fator de recente interesse de pesquisadores sobre o tema ${ }^{6}$, sendo que na década de 90 a preocupação com a violência ganha prioridade nas agendas das organizações internacionais do setor ${ }^{7}$.

O interesse da área de saúde pela violência cresceu em razão de dois fatores: a conscientização cres- cente dos valores da vida e dos direitos de cidadania e as mudanças no perfil de morbidade ${ }^{8}$.

No Brasil, estudos específicos sobre a violência contra idosos no ambiente doméstico, bem como sobre os possíveis fatores a ela associados são bem escassos, embora o conhecimento destas questões seja imprescindível para promoção da saúde, diagnóstico precoce e acompanhamento das vítimas e familiares.

Diante desta perspectiva, o objetivo deste artigo consistiu em identificar a prevalência de violência doméstica contra idosos residentes em uma microrregião do município do Recife/PE bem como investigar os possíveis fatores que podem ter associação com este fenômeno.

\section{Procedimentos metodológicos}

Trata-se de estudo descritivo, de corte transversal, desenvolvido a partir do banco de dados do projeto de pesquisa "O (des) conhecido cenário da violência contra idosos no ambiente doméstico", realizado nos anos de 2009 e 2010 . A pesquisa foi aprovada no Comitê de Ética em Pesquisa do Centro de Ciências da Saúde da Universidade Federal de Pernambuco e foi aprovado.

A investigação foi realizada no Distrito Sanitário IV, centrando-se na microrregião (MR) 4.2, que compreende dois bairros da cidade do Recife/PE e é composta por cinco unidades de saúde da família. Nestas, estão cadastrados 1.366 idosos de acordo Sistema de Informações da Atenção Básica9.

A amostra, que foi selecionada por amostragem sistemática, foi constituída por 274 pessoas idosas (60 anos e mais), de ambos os sexos, não institucionalizadas, residentes na área de abrangência da MR 4.2 e assistidas nas unidades de saúde da área. Neste estudo, foram excluídos os idosos que apresentavam enfermidade ou agravo à saúde que implicasse comprometimento em termos de comunicação.

Para coleta de dados foi utilizado o instrumento para avaliar possíveis situações de violência contra pessoas idosas adotado pelo Ministério da Saúde nos Cadernos de Atenção Básica ${ }^{10}$. Neste, investigava-se a violência "física", "psicológica", "abuso financeiro e econômico". Portanto, foram acrescidas duas perguntas, conforme o Caderno de Atenção Básica no 8 do ano de 2002, para investigar a "violência sexual".

A pesquisa ocorreu entre janeiro e junho de 2010, sendo os dados coletados, principalmente, nos domicílios dos idosos; entretanto, quando 
havia impossibilidade de acesso às suas residências, a coleta era feita nas respectivas unidades de saúde da família. No roteiro da entrevista constavam questões referentes às variáveis biodemográficas, sociodemográficas e as relativas à capacidade funcional do idoso. Para a classificação em relação à capacidade funcional, foram utilizados os instrumentos de avaliação para análise da independência nas atividades instrumentais de vida diária (Escala de Lawton) e atividades de vida diária (Índice de Katz).

O processamento dos dados foi realizado mediante a utilização do EpiInfo para Windows, versão 3.5.1. Já a análise estatística foi através do Programa Stata. Foram realizadas análises univariadas das variáveis, através de frequências absoluta e relativa (variáveis categóricas), média e desvio padrão (no caso da idade), visando à sua descrição e à determinação da maneira pela qual se encontravam distribuídas na população do estudo. Posteriormente foi efetivado o cruzamento entre elas com o objetivo de verificar a existência de possíveis associações, através do teste do quiquadrado de Pearson ou do teste exato de Fisher.

As variáveis que apresentaram um valor $\mathrm{p}$ menor que 0,20 nesses cruzamentos foram selecionadas para compor um modelo de regressão logística multivariada, com o intuito de identificar o possível efeito independente de cada uma delas. Foi adotado como critério para a rejeição da hipótese nula o valor de $\mathrm{p}<0,05$.

$\mathrm{Na}$ fase analítica as variáveis estado conjugal, arranjo familiar e contribuição para o sustento da casa foram estratificadas, com o objetivo de aprimorar a análise estatística e favorecer a discussão com estudos já realizados.

\section{Resultados}

Entre os 274 idosos estudados, $67,15 \%$ pertenciam ao sexo feminino e, a maioria dos entrevistados apresentava entre 60-69 anos (58,03\%). Cerca de $46 \%$ referia ter estudado até três anos. Proporções equivalentes de indivíduos eram casados ou tinham uma união estável e não tinham companheiro(a) (solteiro, viúvo, separado ou divorciado), $44,53 \%$ e $55,47 \%$, respectivamente. Em torno de $47 \%$ dos idosos conviviam no domicílio com duas ou mais pessoas, estando, a maioria, atualmente aposentada $(63,20 \%)$. No que diz respeito à renda mensal, 196 idosos recebiam de um a dois salários mínimos (71,53\%), sendo que $91,88 \%$ contribuíam para o sustento da casa. Os indicadores da capacidade funcional foi avaliado a partir do seu desempenho nas atividades instrumentais de vida diária (AIVD) e atividades básicas de vida diária (AVD). A dependência nas AIVD foi relatada por 39,05\% dos idosos; já a dependência nas AVD foi referida por 4,01\%.

Entre os 274 idosos entrevistados, verificouse a existência de 57 com sinais indicativos de violência, o que corresponde à prevalência de 20,8\% (IC 95\%: 16,0 - 25,6), com pelo menos um dos tipos de violência sofrida pelos idosos em seu ambiente doméstico.

A Tabela 1 apresenta a distribuição da violência contra os idosos no ambiente doméstico, de acordo com as características biodemográficas e sociodemográficas do estudo. A maioria das vítimas de violência foi do sexo feminino $(23,91 \%)$, numa razão de 1,65 mulheres para cada homem. Predominaram as vítimas com idades entre 60 e 69 anos $(22,64 \%)$, e os idosos que nunca estudaram (analfabetos) foram os que mais sofreram violência $(26,15 \%)$, assim como os que não tinham companheiro (solteiro/separado/viúvo) (21,71\%).

Quanto ao arranjo familiar, observou-se que os idosos que moravam com um número maior de coabitantes (6 ou mais moradores) corresponderam a $33,33 \%$ dos casos. Proporções equivalentes foram encontradas em relação à distribuição da situação previdenciária. Ressalte-se ainda que foram mais vitimizados os idosos que recebiam até um salário mínimo $(22,86 \%)$ e os que contribuíam para o sustento da casa $(20,88 \%)$ (Tabela 1$)$.

Em relação à capacidade funcional, constatase que $28(26,17 \%)$ dos idosos dependentes nas AIVD sofreram algum tipo de violência, enquanto as maiores vítimas no que diz respeito às AVD foram os independentes para estas atividades $(20,91 \%)$, só apresentando dois casos entre os idosos dependentes nas AVD (18,18\%) (Tabela 2).

Analisando-se a associação da violência com os fatores demográficos e da capacidade funcional dos idosos, observou-se neste estudo que a prevalência de violência foi mais presente entre os que moravam com um número maior de indivíduos $(33,33 \%)$, ainda que estes valores estejam com significância limítrofe $(p=0,060)$.

Embora com certa ressalva, devido à menor significância estatística, é possível perceber que as prevalências de violência doméstica foram maiores entre as mulheres $(23,91 \%)$ e entre os idosos dependentes para as atividades instrumentais da vida diária $(26,17 \%)$. Quanto às demais variáveis do estudo, não se observaram diferenças estatisticamente significativas entre os grupos (Tabelas 1 e 2). 
Tabela 1. Distribuição da violência doméstica segundo características bio e sociodemográficas (MR4.2, Recife, 2010).

\begin{tabular}{|c|c|c|c|c|c|}
\hline \multirow[b]{3}{*}{ Variáveis do estudo } & \multicolumn{4}{|c|}{ Violência doméstica } & \multirow[b]{3}{*}{ Valor $\mathrm{p}$} \\
\hline & \multicolumn{2}{|c|}{ Sim } & \multicolumn{2}{|c|}{ Não } & \\
\hline & $\mathbf{N}$ & $\%$ & $\mathbf{N}$ & $\%$ & \\
\hline Sexo & & & & & $0,070^{*}$ \\
\hline Masculino & 13 & 14,44 & 77 & 85,56 & \\
\hline Feminino & 44 & 23,91 & 140 & 76,90 & \\
\hline Faixa Etária & & & & & $0,552^{* *}$ \\
\hline 60 a 69 anos & 36 & 22,64 & 123 & 77,36 & \\
\hline 70 a 79 anos & 17 & 20,00 & 68 & 80,00 & \\
\hline 80 e mais & 4 & 13,33 & 26 & 86,67 & \\
\hline Escolaridade (em anos de estudo) & & & & & $0,459^{*}$ \\
\hline Nunca estudou/analfabeto & 17 & 26,15 & 48 & 73,85 & \\
\hline Até 3 anos & 23 & 18,40 & 102 & 81,60 & \\
\hline Entre 4 e 10 anos & 17 & 20,73 & 65 & 79,27 & \\
\hline Estado conjugal & & & & & $0,680^{*}$ \\
\hline Casado (a) ou união estável & 24 & 19,67 & 98 & 80,33 & \\
\hline Solteiro/separado/viúvo & 33 & 21,71 & 119 & 78,29 & \\
\hline Arranjo Familiar & & & & & $0,060^{*}$ \\
\hline Até 5 moradores & 46 & 19,09 & 194 & 80,81 & \\
\hline 6 ou mais moradores & 11 & 33,33 & 22 & 66,67 & \\
\hline Situação previdenciária ${ }^{* * *}$ & & & & & $0,758^{*}$ \\
\hline Aposentado(a) & 33 & 19,41 & 137 & 80,59 & \\
\hline Pensionista/benefício & 13 & 23,21 & 43 & 76,79 & \\
\hline Não é aposentado(a) & 10 & 23,26 & 33 & 77,52 & \\
\hline Renda & & & & & $0,876^{* *}$ \\
\hline Menos de um salário mínimo & 8 & 22,86 & 27 & 77,14 & \\
\hline De um a dois salários mínimos & 42 & 21,43 & 154 & 78,57 & \\
\hline De dois a quatro salários & 7 & 17,95 & 32 & 82,05 & \\
\hline Mais de quatro salários & 0 & 0 & 4 & 100,00 & \\
\hline Contribuição para o sustento da casa ${ }^{* * *}$ & & & & & $1,000^{* *}$ \\
\hline Sim & 52 & 20,88 & 197 & 79,12 & \\
\hline Não & 4 & 18,18 & 18 & 81,81 & \\
\hline
\end{tabular}

* Teste do Qui-quadrado de Pearson; ** Teste Exato de Fisher; ${ }^{* * *}$ O número total variou em função da exclusão dos casos com informação imprecisa.

Tabela 2. Distribuição da violência doméstica em relação à capacidade funcional, segundo as Atividades Instrumentais da Vida Diária e Atividades da Vida Diária. (MR4.2, Recife, 2010).

\begin{tabular}{|c|c|c|c|c|c|}
\hline \multirow{3}{*}{$\begin{array}{c}\text { Capacidade } \\
\text { Funcional }\end{array}$} & \multicolumn{4}{|c|}{ Violência doméstica } & \multirow{3}{*}{ Valor $\mathrm{p}$} \\
\hline & \multicolumn{2}{|c|}{ Sim } & \multicolumn{2}{|c|}{ Não } & \\
\hline & $\mathbf{N}$ & $\%$ & $\mathbf{N}$ & $\%$ & \\
\hline AIVD & & & & & $0,080^{* *}$ \\
\hline Independente & 29 & 17,37 & 138 & 82,63 & \\
\hline Dependente $^{*}$ & 28 & 26,17 & 79 & 73,83 & \\
\hline AVD & & & & & $1,000^{* *}$ \\
\hline Independente & 55 & 20,91 & 208 & 79,09 & \\
\hline Dependente $^{*}$ & 2 & 18,18 & 9 & 81,82 & \\
\hline
\end{tabular}

* Para esta análise, foram considerados dependentes os idosos que possuíam dependência parcial ou total; ${ }^{* *}$ Teste do Qui-quadrado de Pearson.
As variáveis associadas à violência doméstica contra os idosos que apresentaram $\mathrm{p}<0,20$ foram sexo e arranjo familiar (Tabela 1) e Atividades Instrumentais da Vida Diária - AIVD (Tabela 2). Com essas três variáveis foi construído um modelo logístico multivariado para avaliar o efeito individual dessas variáveis sobre a violência contra o idoso em seu ambiente doméstico.

A Tabela 3 apresenta os resultados do ajuste deste modelo. Apenas as variáveis sexo e arranjo familiar ficaram significativamente associadas $\left(\mathrm{p} \leq^{\wedge} 0,05\right)$ com a violência doméstica contra os idosos. Os resultados mostram que houve evidência de maior violência contra os idosos que coabitavam com seis ou mais moradores do que entre os que viviam com até cinco moradores. 
Tabela 3. Resultado do ajuste do modelo de regressão logística múltipla.

\begin{tabular}{|c|c|c|c|c|c|c|}
\hline & Amostra & $\begin{array}{c}\text { Prevalência de } \\
\text { Violência Doméstica } \\
\%\end{array}$ & $\mathrm{OR}_{\mathrm{b}}(\mathrm{IC} 95 \%)$ & Valor p & $\mathrm{OR}_{\mathrm{a}}(\mathrm{IC} 95 \%)$ & Valor $\mathrm{p}$ \\
\hline Sexo & & & & 0,07 & & 0,05 \\
\hline Masculino & 90 & 14,4 & 1,0 & & 1,0 & \\
\hline Feminino & 184 & 23,9 & $1,9(0,9-3,7)$ & & $2,0(1,0-4,0)$ & \\
\hline Arranjo familiar & & & & 0,06 & & 0,04 \\
\hline Até 5 moradores & 240 & 19,2 & 1,0 & & 1,0 & \\
\hline 6 ou mais moradores & 33 & 33,3 & $2,1(1,0-4,7)$ & & $2,4(1,0-5,3)$ & \\
\hline
\end{tabular}

" Para esta análise, foram considerados dependentes os idosos que possuíam dependência parcial ou total; "* Teste do Qui-quadrado de Pearson.

Além disso, as mulheres estariam mais sujeitas à possibilidade de ocorrer violência dentro do ambiente doméstico em relação aos homens.

\section{Discussão}

De acordo com Reichenheim et al. ${ }^{11}$, apesar de o tema vir crescendo nos últimos anos e estudos sugerirem que o fenômeno tem grande expressividade, o direcionamento ao grupo idoso, é mais recente, não surpreendendo uma carência de informação. Contribui para isto a própria complexidade da temática e a dificuldade recorrente no seu reconhecimento.

A prevalência de violência contra idosos no ambiente doméstico no presente trabalho foi superior à observada em estudos desenvolvidos nos Estados Unidos, Canadá e na Holanda ${ }^{5}$.

Em um estudo descritivo para estimar a prevalência de maus-tratos contra idosos no município de Camaragibe/PE, os autores encontraram uma prevalência de $21 \%$ destacando-se que, quando especificado para violência doméstica, este dado corresponde a $62,1 \%^{12}$.

Investigando uma população cadastrada no Programa Médico da Família, em Niterói (RJ), e com o objetivo de estimar a prevalência de violência física, foi estimado que $10,1 \%$ dos idosos entrevistados sofreram violência no ano anterior à entrevista ${ }^{13}$. Em publicação mais recente, Apratto Júnior ${ }^{14}$, com a população de mesmas características, estimou a prevalência da violência psicológica em $43,2 \%$.

Entretanto, salienta-se que Abath ${ }^{15}$ compartilha com a visão de diversos autores no que diz respeito à dificuldade de comparar as prevalências nas pesquisas que envolvem violência contra os idosos, diante das diferentes definições conceituais adotadas, características das amostras investigadas e das formas de identificação das situações de violência.

Diversos estudos têm descrito que as mulheres são mais abusadas do que os homens. Isto, possivelmente, decorre quando a questão de gênero se alia ao fato de as mulheres estarem em situação de maior vulnerabilidade do que os homens. No presente trabalho, a violência foi mais frequente no sexo feminino.

Conforme pesquisas, há diferenças nas estimativas de violência quanto ao local de ocorrência. No ambiente doméstico as principais vítimas são do sexo feminino $0^{6,12,16}$.

Nas denúncias recebidas pela Voz do Cidadão, notou-se que, no que se refere à violência doméstica, as mulheres são mais vitimizadas $(68 \%)$ do que os homens (25\%), não tendo sido registrado o sexo em $7 \%$ das denúncias. Quanto ao tipo de violência, houve predominância da agressão física (28), financeira (25) e psicológica (23) e, além disso, o registro de duas denúncias de violência sexual entre o sexo feminino. Já entre os homens, o maior número de denúncias foi de abandono (8), sendo acompanhada pelos maus tratos físicos (7) e financeiros (7) ${ }^{17}$.

Corroborando esses achados, Melo et al. ${ }^{12}$, quando especificaram o local de ocorrência, observaram a associação entre o sexo e a violência doméstica, estimando que $78 \%$ das vítimas eram as mulheres. Moraes et $\mathrm{al}^{1{ }^{13}}$, apesar de não encontrarem associação, identificaram que $61,3 \%$ das vítimas de violência física eram do sexo feminino.

Entretanto, contrariando tais achados, Gaioli e Rodrigues ${ }^{18}$ informaram que, na análise dos laudos de sua pesquisa, ocorreu maior proporção $(58,6 \%)$ de homens vítimas de violência no ambiente doméstico.

Ainda que pareça consensual entre alguns estudos a relação da associação entre violência contra os idosos e o sexo, autores ${ }^{16}$ salientam o fato 
de que pesquisas desenvolvidas no Canadá, nos Países Baixos e nos Estados Unidos, realizadas nos últimos anos, revelaram que não há diferenças significativas nas taxas prevalentes de abusos por sexo. Isto pode de alguma forma, ser justificado pelas diferenças culturais existentes entre esses países.

No que se refere à idade, na maioria dos casos, a violência ocorreu na faixa etária entre 60 e 69 anos (22,64\%), não sendo observada significância estatística para esta variável. Ainda que a literatura aponte que as maiores vítimas são idosos mais velhos, principalmente devido às limitações funcionais e cognitivas, nos estudos onde foi pesquisada esta variável ocorrem algumas disparidades em relação à predominância deste evento.

Estes resultados aproximaram-se daqueles encontrados em outros estudos nacionais, como os de Abath ${ }^{15}$, Apratto Júnior ${ }^{14}$ e Moraes et al. ${ }^{13}$. Abath ${ }^{15}$ encontrou uma frequência de $71,6 \%$ de violência doméstica do tipo física contra os idosos submetidos a exame de corpo de delito no Instituto Médico Legal na cidade do Recife. Embora a autora ressalte que, por se tratar de uma pesquisa em serviço especializado, esta faixa etária, por ter mais condições físicas e mentais, possui maior capacidade de ir aos órgãos de denúncia e, por isso, tende a apresentar valores mais elevados.

Já as prevalências na publicação de Apratto Júnior $^{14}$ foram de $46,4 \%$ (no caso da violência psicológica) e 13,4\% (no caso da violência física), e na de Moraes et al. ${ }^{13}$ foram encontrados os percentuais de $11,7 \%$ e 9,4\% para os casos de violência física menor e grave, respectivamente, para a mesma faixa etária. Vale salientar que estes valores corroboram os dados encontrados nesta pesquisa e que apresentam o desenho do estudo e as populações bem similares à analisada.

Nesse sentido, foi discordante da encontrada por Souza et al. ${ }^{19}$, onde se registrou uma maior frequência entre os 70-79 anos, sendo de 35,1\% no Núcleo de Atendimento a Pessoa Idosa e 31,3\% na Delegacia do Idoso. Souza et al. ${ }^{16}$ observaram maiores percentuais de vitimização (37\%) na faixa etária entre 71 e 80 anos de idade. Estas diferenças podem ser justificadas em virtude de terem sido realizadas em serviços especializados de atendimento às pessoas vítimas de violência, tendendo, desta forma, a apresentar valores diferenciados quando comparados com a presente pesquisa.

Iniciando a análise das variáveis sociodemográficas, o analfabetismo entre os idosos nos re- sultados da nossa investigação não se mostrou como fator associado à violência doméstica, ainda que tenha tido uma maior proporção de vítimas entre os que nunca estudaram. Além disso, revelou-se um percentual maior de vítimas entre os que possuíam entre quatro e dez anos de estudo $(20,73 \%)$ do que entre os que tinham até três anos de estudo (18,40\%). Pelo exposto, mesmo que haja frequências maiores entre aqueles que não têm nenhuma escolaridade, não se pode presumir que idosos com menor nível de instrução sejam considerados mais favoráveis à violência.

Esses achados apoiam os de Moraes et al. ${ }^{13}$ que, em levantamento, verificaram que a ocorrência da violência física grave foi cerca de três vezes mais comum entre os idosos com maior escolaridade em comparação aos que tinham até três anos de estudo. Já entre aqueles que foram vítimas de violência física menor, demonstrouse que não há esta associação, pelo fato de os idosos com até três anos de estudo apresentarem $9,4 \%$, enquanto entre 4 e 10 anos $6,3 \%$ e com 11 anos ou mais de estudo 6,7\%.

Nesse sentido, ao contrário do que se esperaria, a violência doméstica não variou segundo o grau de escolaridade dos idosos. Apesar de os autores relatarem que são maiores vítimas aqueles com menor grau de instrução, Moraes et al. ${ }^{13}$ apontam que os que possuem menor escolaridade podem apresentar uma dependência financeira ou em atividades cotidiana maior em relação aos cuidadores. Entretanto, o medo de perder a fonte de carinho e afeto, bem como o receio de represálias, pode impedir a declaração de violência entre eles.

Apesar dos valores bem similares em relação ao estado conjugal, não evidenciando significância estatística entre a violência doméstica e esta variável, os maiores percentuais de vítimas entre aqueles que não têm companheiro (solteiros, separados ou viúvos) contradizem os achados da literatura, que assinalam que as principais vítimas são as que possuem companheiro.

De certa forma, isto pode ser explicado pelo fato de que, embora convivam com outro membro na unidade, a literatura aponta que os maiores agressores no contexto doméstico são os filhos dos idosos, seguidos do companheiro ${ }^{18,20,21}$ ou genros/ noras $^{22}$. Somado a isso, teria que se considerar especificidades deste companheiro, uma vez que, como já destacado, no surgimento da violência os fatores parecem estar inter-relacionados.

Utilizando-se uma categorização diferente desta pesquisa, Apratto Júnior ${ }^{14}$ não encontrou associação entre o estado civil e a violência domésti- 
ca; contudo, apontaram que as vítimas mais frequentes estavam no grupo dos casados quando comparados os viúvos, tanto para a violência psicológica quanto para a violência física grave e total, assim como Gaioli e Rodrigues ${ }^{18}$, que apontaram as maiores vítimas entre os casados ou amasiados $(46,4 \%)$, e Moraes et al. ${ }^{13}$, que registraram que foram mais vitimizados os idosos que tinham companheiro, embora a classificação esteja bem diferenciada da presente pesquisa.

Em relação ao número de moradores, este estudo confirma os resultados encontrados em outras pesquisas. Verifica-se uma importante relação do arranjo familiar com a violência doméstica, pois a prevalência de violência foi maior entre os que moravam com um número maior de habitantes.

$\mathrm{Na}$ análise, percebe-se a consonância com diversos estudos que afirmam que os idosos que convivem com maior número de pessoas no domicílio foram mais vitimizados ${ }^{13,14,21,23}$.

Com base na verificação dos inquéritos populacionais realizados em Niterói (RJ) com população de idosos também cadastrados em unidades de saúde da família, constatou-se associação entre o número de moradores na residência e a presença de violência doméstica. Com categorização idêntica à do presente estudo, Moraes et al..$^{13}$ observaram que $19,2 \%$ dos idosos que conviviam com seis ou mais moradores eram as maiores vítimas de violência física, assim como Apratto Júnior ${ }^{14}$ demonstrou que, independente do tipo de violência sofrida - psicológica ou física - as prevalências eram maiores entre os idosos que coabitavam em ambiente com um número maior de residentes, $61,5 \%$ e $19,2 \%$, respectivamente.

Por isso, Fonseca et al. ${ }^{3}$ argumentam que o fato de os idosos conviverem na mesma unidade doméstica com diferentes gerações pode ser encarada como um potencial causador de conflitos intrafamiliares. Assim, o convívio plurigeracional não pode ser visto como garantia de velhice bem sucedida, nem mesmo sinal de relações mais amistosas entre as sucessivas gerações.

Pelo fato de a família ser o lócus privilegiado de moradia e cuidado desses idosos, é preciso investir na sua competência para abrigá-lo com respeito e dignidade ${ }^{22}$. Por esta razão é que Grossi et al. ${ }^{21}$ citaram que a responsabilidade dos familiares não deve ser a única alternativa de cuidado para o idoso, sendo necessária a contribuição da sociedade, independentemente de estes receberem ou não o apoio de suas famílias.

Por outro lado, Melo et al. ${ }^{12}$ não encontraram diferença na prevalência de violência nos ido- sos que viviam com pessoas da mesma geração ou entre os que coabitavam em ambientes multigeracionais. Todavia, não pode ser comparado com a presente pesquisa, tendo em vista que a categorização foi distinta da empregada neste estudo.

Quanto à situação previdenciária, foi constatado que os valores apresentaram-se bem semelhantes em relação à prevalência de violência entre as categorias, não demonstrando nenhuma relação de associação. Ressalte-se que na literatura consultada não foi encontrada análise abordando esta variável, tornando-se a comparação inexequível.

Analisando-se a renda do idoso pode-se verificar que a ocorrência de violência foi maior entre os que recebiam menos de um salário mínimo, e foi diminuindo à medida que aumentavam os valores recebidos pelo idoso, ainda que estas ocorrências estejam com valores bem similares e, portanto, não evidenciam associação do evento com a variável. É válido enfatizar que na literatura consultada não foram encontrados relatos de pesquisas que tenham investigado a renda do idoso nos estudos sobre violência doméstica enquanto fator associado, podendo ser possível a elaboração de hipóteses explicativas.

Um dos fortes motivos da violência podem ser as questões de ordem financeira, uma vez que se pode supor que, quanto maior a renda do idoso, melhores condições de vida ele terá e maiores serão as possibilidades de contribuição desta renda no sustento da família.

Em relação à variável contribuição para o sustento da casa, não foram encontradas pesquisas que abordassem este tópico, não sendo viável a comparação, apenas a elaboração de algumas hipóteses explicativas, embora haja menções de que tanto o idoso sustentado por um cuidador quanto a relação inversa apresentamse como potencial de risco para a violência ${ }^{12}$.

Araújo et al..$^{24}$ e Grossi et al. ${ }^{21}$ identificaram que a maioria das pessoas que convivem com os idosos depende de sua renda e isto, em geral, é favorecido pela política dos benefícios previdenciários. Segundo os autores, este fato é decorrente do aumento do desemprego e do número de divórcios que propicia o retorno dos filhos adultos para a casa de seus pais, desenvolvendo uma dependência emocional e financeira, o que pode levar ao abuso financeiro ou material do idoso.

Com base nisso é que Abath ${ }^{15}$ pontua que nas residências onde os idosos não são responsáveis pelo sustento predominam os mais velhos, mais pobres, com piores condições de saúde e 
menor capacidade funcional, quando comparadas às dos idosos chefes dos domicílios.

Considerando a análise da capacidade funcional, em informe específico do Ministério da Saúde os autores apontam que as avaliações funcionais dão ênfase às Atividades de Vida Diária ${ }^{10}$. $\mathrm{Na}$ Escala de Katz pode-se medir a habilidade no desempenho das atividades cotidianas de forma independente, e o instrumento de Lawton permite avaliar as Atividades Instrumentais de Vida Diária (AIVD), consideradas mais complexas e cuja independência para desempenho está diretamente relacionada com a capacidade de vida comunitária ${ }^{10,25}$.

Considerando esta informação, os autores expõem que a perda de habilidades funcionais que ocorrem fisiologicamente no processo de envelhecimento acontece de forma hierárquica, sendo, portanto, direcionada das AIVD para as $\mathrm{AVD}^{25}$.

Nesse sentido, no que diz respeito à capacidade funcional, à medida que aumenta o grau de dependência, maior é a chance de o idoso ser vítima de violência. Os idosos que necessitam de ajuda para sobreviver, principalmente devido a problemas de saúde, apresentam maior risco de sofrer algum tipo de abuso ou mau trato, quando não há entendimento entre o idoso e a família ${ }^{21}$. Isto corrobora com a visão de Saliba et al. ${ }^{26}$, que afirmam que com o avançar da idade aumentam as situações de vulnerabilidade, o que gera maior necessidade de cuidados, criando uma situação de dependência. Isto favorece a ocorrência de abusos entre os idosos mais dependentes.

Embora Melo et al. ${ }^{12}$ afirmem que a maior dependência foi considerada inicialmente um fator de risco, estudos posteriores mostraram que os idosos vitimizados não eram mais dependentes do que aqueles que não sofriam maus tratos, sendo, conforme os autores, talvez mais capazes, principalmente nos casos de violência física e verbal.

Pelo exposto, a distribuição da violência doméstica em relação à capacidade funcional variou entre as variáveis de Atividades Instrumentais de Vida Diária (AIVD) e Atividades Básicas de Vida Diária (AVD). Quando analisada a AVD, notou-se que a prevalência de violência foi ligeiramente maior entre os idosos que não tinham dependência; quando analisada em relação à AIVD, porém, ficou demonstrado que os idosos que eram dependentes eram os mais vitimizados (26\%), e que, embora com certa ressalva devido à menor significância estatística, a dependência nas atividades mais complexas pode ser conside- rada um fator associado à violência contra idosos no ambiente doméstico.

Isto pode ser justificado, em parte, pelo fato de que a perda de autonomia física e/ou cognitiva, levando à demanda de realização das atividades cotidianas e instrumentais por outras pessoas, cria, consequentemente, uma relação de "desempoderamento" (grifo do autor) e subordinação do idoso ${ }^{13}$.

Destaca-se que a violência cometida contra os idosos possui, de acordo com a literatura pesquisada, inúmeros fatores associados que, pela possibilidade de inter-relação entre as variáveis, podem ocasionar confundimento em termos de associação. Nesse sentido, apesar de não ter havido referências que abordassem essa apreciação, considerou-se importante realizar a análise multivariada com o sentido de identificar o efeito independente da associação.

Portanto, as evidências de que os idosos que coabitavam em ambientes com um número maior de pessoas (6 ou mais moradores) é significativamente associada apontam que a convivência multigeracional representa um indício de que os idosos que convivem com mais indivíduos no mesmo espaço físico podem ser mais vitimizados do que aqueles que convivem com até cinco moradores. Além disto, pode-se verificar que as mulheres idosas estariam mais sujeitas a serem vítimas de violência no ambiente doméstico do que os homens, o que é relatado pela maior parte das publicações que abordam o assunto, como já explicitado anteriormente.

\section{Considerações finais}

A identificação das ocorrências e os fatores associados à violência contra os idosos no ambiente doméstico é o primeiro passo para o estudo do fenômeno que tem se tornado grande problema de Saúde Pública.

Ainda que o presente estudo tenha buscado a associação do fenômeno da violência doméstica, com o desenho do estudo não é possível estabelecer uma relação de causa e efeito entre estes fatores, o que sugere que o evento tenha sido causado pelos fatores acima descritos.

A relação entre o perfil dos idosos estudados e as variáveis analisadas é apoiada por estudos já relacionados sobre esta temática. Apesar disso, salienta-se que ainda são muitas as questões a serem respondidas tornando fundamental a ampliação de investigações nesta área. 


\section{Colaboradores}

AM Duque, MCC Leal, APO Marques, FMV Eskinazi e AM Duque participaram igualmente de todas as etapas de elaboração do artigo.

\section{Referências}

1. Brasil. Ministério da Saúde (MS). Informe de situação e tendências: demografia e saúde. Rede Interagencial de Informações para Saúde. Brasília: MS; 2009.

2. Veras R. Envelhecimento populacional contemporâneo: demandas, desafios e inovações. Rev Saude Publica 2009; 43(3):548-554.

3. Fonseca MM, Gonçalves, HS. Violência contra o idoso: suportes legais para a intervenção. Interação em Psicologia 2003; 7(2):121-128.

4. Bruno LMSM. Considerações sobre o abuso financeiro de pessoas idosas e a dinâmica das relações familiares. Textos Envelhecimento 2005; 8(2):1-15.

5. Espíndola CR, Blay SL. Prevalência de maus tratos na terceira idade: revisão sistemática. Rev Saude Publica 2007; 41(2):301-306.

6. Minayo MC. Violência contra idosos: relevância para um velho problema. Cad Saude Publica 2003; 19(3):783-791.

7. Minayo MC. A violência social sob a perspectiva da saúde pública. Cad Saude Publica 1994; 10(Supl. 1):7-18.

8. Machado L, Queiroz Z. Negligência e maus-tratos. In: Freitas EV, Py L, Neri AL, Cançado FAXC, Gorsoni ML, Doll J, organizadores. Tratado de Geriatria e Gerontologia. Rio de Janeiro: Guanabara Koogan; 2006. p. 1152-1159.

9. Prefeitura do Recife. Sistema de Informações da Atenção Básica; 2009.

10. Brasil. Ministério da Saúde (MS). Secretaria de Atenção a Saúde, Departamento de Atenção Básica. Envelhecimento e Saúde da pessoa idosa. Brasília: MS; 2006. (Cadernos de Atenção Básica, nº19)

11. Reichenheim ME, Paixão Júnior CM, Moraes CL. Adaptação transcultural para o português (Brasil) do instrumento Hwalek-Sengstock Elder Abuse Screening Test (HS/EAST) utilizado para identificar risco de violência contra o idoso. Cad Saude Publica 2008; 24(8):1801-1813.

12. Melo VL, Cunha JOC, Falbo Neto GH. Maus-tratos contra idosos no município de Camaragibe, Pernambuco. Rev. Bras. Saúde Mater Infant 2006; 6(Supl. 1):543-548.

13. Moraes CL, Apratto Júnior PC, Reichenheim ME. Rompendo o silêncio e suas barreiras: um inquérito domiciliar sobre a violência doméstica contra idosos em área de abrangência do Programa Médico da Família de Niterói, Rio de Janeiro, Brasil. Cad Saude Publica 2008; 24(10):2289-2300.

14. Apratto Júnior PC. A violência doméstica contra os idosos nas áreas de abrangência do Programa de Saúde da Família de Niterói (RJ, Brasil). Cien Saude Colet 2010; 15(6):2983-2995.

15. Abath MB. Violência contra a pessoa idosa: o que revelam os laudos periciais do Instituto de Medicina Legal [dissertação]. Recife (PE): Universidade Federal de Pernambuco; 2009.

16. Souza JAV, Freitas MC, Queiroz TA. Violência contra idosos: análise documental. Rev. bras. enferm. 2007; 60(3):268-272. 
17. Pasinato MT, Camarano AA, Machado L. Idosos vítimas de maus-tratos domésticos: estudo exploratório das informações levantadas nos serviços de denúncia. In: Anais do XIV Encontro Nacional de Estudos Populacionais; 2004; Caxambú. [documento na Internet]. 2006 [acessado $22 \mathrm{dez}$ 2010]. [34p] Disponível em: http:// ipea.gov.br/pub/td/2006/td_1200.pdf

18. Gaioli CCLO, Rodrigues RAP. Ocorrência de maus-tratos em idosos no domicílio. Rev Latino-Am Enfermagem [periódico na internet]. 2008 [acessado 25 abr 2011]; 16(3): [6p.]. Disponível em: http://www.scielo.br/pdf/ rlae/v16n3/pt_21.pdf

19. Souza ER, Ribeiro AP, Atie S, Souza AC, Marques CC. Rede de proteção aos idosos do Rio de Janeiro: um direito a ser conquistado. Cien Saude Colet 2008; 13(4):1153-1163.

20. Brasil. Ministério da Justiça. Subsecretaria de Direitos Humanos. Plano de Ação para o Enfrentamento da Violência Contra a Pessoa Idosa. Brasília: Subsecretaria de Direitos Humanos; 2005.

21. Grossi PK, Souza MR. Os idosos e a violência invisibilizada na família. Textos e Contextos 2003; 2(1):1-14.

22. Brasil. Ministério da Saúde. Temático: prevenção de violência e cultura de paz. Brasília: Organização Pan-Americana de Saúde; 2008. 60p. (Série painel de indicadores do SUS, v.3, n.5).

23. Souza AS, Meira EC, Neri IG, Silva JA, Gonçalves LHT. Fatores de risco de maus-tratos ao idoso na relação idoso/cuidador em convivência intrafamiliar. Textos Envelhecimento 2004; 7(2):1-15.

24. Araújo TCN, Alves MIC. Perfil da população idosa no Brasil. Textos Envelhecimento 2000; 3(3):7-19.

25. Lebrão ML, Laurenti R. Saúde, bem-estar e envelhecimento: o estudo SABE no município de São Paulo. Rev. Bras. Epidemiol. 2005; 8(2):127-141.

26. Saliba O, Garbin CAS, Garbin AJI, Dossi AP. Responsabilidade do profissional de saúde sobre a notificação de casos de violência doméstica. Rev Saude Publica 2007; 41(3):472-477.

Artigo apresentado em 08/03/2012

Aprovado em 12/03/2012

Versão final apresentada em 29/03/2012 